\title{
Cryptotanshinone interferes with chondrocyte apoptosis in osteoarthritis by inhibiting the expression of miR-574-5p
}

\author{
SONGTAO YUE ${ }^{1}$, XIAOCHUAN $\mathrm{SU}^{2}$, JUNYAN TENG ${ }^{2}$, JIANGYI WANG $^{1}$ and MALONG GUO ${ }^{1}$ \\ ${ }^{1}$ Department of Osteoarthrosis, ${ }^{2}$ Health Management Center, Luoyang Orthopedic-Traumatological Hospital of \\ Henan Province (Henan Provincial Orthopedic Hospital), Zhengzhou, Henan 450000, P.R. China
}

Received September 30, 2020; Accepted February 26, 2021

DOI: $10.3892 / \mathrm{mmr} .2021 .12063$

\begin{abstract}
Chondrocyte apoptosis is an important factor in the development and progression of osteoarthritis (OA). Cryptotanshinone (CTS) can inhibit chondrocyte apoptosis, but the specific mechanism remains unknown. The aim of the present study was to explore how CTS may affect chondrocyte apoptosis. Reverse transcription-quantitative PCR and western blotting were used to validate microRNA (miR)-574-5p, YY1-associated factor 2 (YAF2), Bcl-2 and Bax expression levels. H\&E, Safranin O and TUNEL staining assays were used to evaluate the apoptosis of arthritic chondrocytes in vivo. A Cell Counting Kit-8 assay and flow cytometry were performed to detect cell proliferation and apoptosis of chondrocytes in vitro. The methylation level of the miR-574-5p promoter was measured via methylation specific PCR. The degree of chondrocyte apoptosis and the expression levels of YAF2 and Bcl-2 were decreased in the mice with OA, and were increased in the OA + CTS mice, while the expression levels of miR-574-5p and Bax showed opposite changes. Furthermore, the degree of chondrocyte apoptosis and the expression levels of the aforementioned key factors in chondrocytes were consistent with those observed in vivo. The methylation degree of the miR-574-5p promoter was increased by the addition of CTS, and was reduced after the addition of a methylation inhibitor, 5-aza-CdR, indicating that CTS could regulate the methylation of miR-574-5p promoter. The present study suggested that CTS could downregulate the expression of miR-574-5p by regulating its methylation, and thus, could improve YAF2 expression and affect chondrocyte apoptosis.
\end{abstract}

Correspondence to: Dr Malong Guo, Department of Osteoarthrosis, Luoyang Orthopedic-Traumatological Hospital of Henan Province (Henan Provincial Orthopedic Hospital), 100 Yongping Road, Zhengzhou, Henan 450000, P.R. China

E-mail:m16542@126.com

Key words: cryptotanshinone, chondrocyte apoptosis, osteoarthritis, microRNA-574-5p, YY1-associated factor 2

\section{Introduction}

Osteoarthritis (OA) is caused by the imbalance of the decomposition and anabolic metabolism among chondrocytes, extracellular matrix (ECM) and subchondral bone. Moreover, it is manifested as pain and inflammation in the affected joints, and in severe cases, leads to restricted or even disabled joint activity $(1,2)$. OA is a common disease worldwide, and with the aging of the population, the incidence of OA increases year by year $(3,4)$. OA not only harms the health of patients and affects their quality of life, it also causes a heavy economic burden on families and society (5).

The main pathological feature of OA is the significantly increased apoptotic rate of articular chondrocytes (6). Apoptosis is a type of programmed cell death, which is initiated under the action of in vivo or in vitro factors, such as hypoxia and nutrient deprivation, eventually leading to increased expression levels of apoptosis-related proteins and the occurrence of apoptosis (7).

Chondrocytes, the only cell component existing in articular cartilage, are mainly responsible for the synthesis and renewal of ECM, but also serve an irreplaceable role in maintaining the normal structure and physiological function of articular cartilage (8). Since normal chondrocytes are a necessary for maintaining the stability of ECM, chondrocyte apoptosis is a key factor in the degeneration of articular cartilage in OA, and the proportion of apoptosis is highly consistent with the destruction of articular cartilage (9). Therefore, it is particularly important to promote chondrocyte proliferation and inhibit its apoptosis, which is a key link in alleviating OA.

Our previous research has confirmed that Zhuanggu huoxue Decoction can promote the anabolism of cartilage matrix in rabbits with OA, including increasing the content of glucuronic acid in cartilage, reducing the moisture content of cartilage and preventing and slowing the degeneration of cartilage in OA (10), indicating that Zhuanggu huoxue Decoction has great application value in OA. However, due to the multi-component, multi-target and integrated characteristics of traditional Chinese medicine (TCM), its effective components and specific mechanisms are unknown, which limits its wide application to some extent (11). Therefore, the present study will further analyze the possible effective components of Zhuanggu huoxue Decoction and clarify its mechanisms. 
Zhuanggu huoxue Decoction is a clinical prescription for the treatment of OA using Pingle Village Guo's bone setting, which is composed of Radix rehmanniae preparata, Chinese yam, Achyranthes bidentata, Eucommia ulmoides, Asiatic cornelian cherry fruit, Salvia miltiorrhiza, Eupolyphaga sinensis and Carthami flos, amongst other ingredients (12). Among these factors, cryptotanshinone (CTS) is the most effective water-soluble component in the pharmacological effect of Salva miltiorrhiza, which has been shown to have anti-inflammatory, anti-tumor and cell activity protective effects $(13,14)$. CTS was confirmed to effectively inhibit IL-1 $\beta$-induced secretion of inflammatory factors (IL-6 and IL-8) in OA synovial cells and reduce MMP-1 expression (15). Moreover, CTS has been reported to alleviate the development of OA in mice by reducing the expression levels of MMP-13 and aggrecanases (such as ADAMTS like 5) that can induce chondrocyte degradation (15). CTS has also demonstrated protective effect on chondrocytes, but the specific targets remain unknown, which is also the focus of the present study.

\section{Materials and methods}

Bioinformatics analysis. The microarray GSE93008 dataset (16) was downloaded from the Gene Expression Omnibus (GEO) database (https://www.ncbi.nlm.nih. gov/pubmed). This dataset comprised three normal controls and three osteoarthritis samples. The relevant genes in the samples were analyzed using GEO2R (http://www.ncbi.nlm. nih.gov/geo/geo2r) and $\mathrm{R}$ language program (4.0.3). The screening conditions were adjusted $\mathrm{P}<0.05$ and $\mid \log \mathrm{FCl} \geq 1$.

OA mouse model. A total of 12 male C57BL/6 mice (age, 12 weeks; weight, 20-25 g) were provided by Junke Biological Co., Ltd., and housed in ZK-SLJ mouse cages (Henan Zhike Hongrun Environmental Protection Technology Co., Ltd.) at $20^{\circ} \mathrm{C}$ with $50 \%$ humidity and a 12 -h light/dark cycle. The animals had unlimited access to a standard rodent chow and water, and adapted for $\geq 7$ days before the experiment. The mice were randomly divided into three groups of 12 mice each: i) Sham group, the mice were injected with $20 \mu 10.9 \%$ normal saline; ii) OA model group, $20 \mu 10.9 \%$ normal saline containing $0.6 \mathrm{mg}$ monosodium iodoacetate (MIA; AIKE Regent) was injected into the knee joint and after 14 days, the mice were gavaged with $0.5 \%$ sodium carboxymethyl cellulose; and iii) OA + CTS group, CTS (MedChemExpress) was dissolved in $0.5 \%$ sodium carboxymethyl cellulose (Sangon Biotech Co., Ltd.) to form an oral suspension and then gavaged using a syringe after 14 days of MIA injection $(10 \mathrm{mg} / \mathrm{kg} /$ day $)$ until 1 day before death. After performing the Von Frey test, the mice were sacrificed by cervical dislocation, and then the knee tissues were collected. The knee tissues of 6 mice were stained, and the expression levels of related genes in the other 6 mouse knee tissues were detected via western blotting and reverse transcription-quantitative (RT-q) PCR, amongst other techniques. All animal procedures were approved by Luoyang Orthopedic-Traumatological Hospital of Henan Province (Henan Provincial Orthopedic Hospital) and complied with the Institutional Animal Care and Use Committee.
Cell cultures and treatments. Isolation and culture of chondrocytes was performed as described previously (17). The knee joints were collected from male C57BL/6 mice (age, 4 days; weight, <12 g; Junke Biological Co., Ltd.; housing conditions as previously described), cleaned with sterile PBS at $4^{\circ} \mathrm{C}$ and cut into pieces. After digestion with $0.25 \%$ trypsin (Thermo Fisher Scientific, Inc.) in an incubator at $37^{\circ} \mathrm{C}$ for $30 \mathrm{~min}$, the knee joints were incubated with $0.2 \%$ collagenase type II (Thermo Fisher Scientific, Inc.) at $37^{\circ} \mathrm{C}$ for another $24 \mathrm{~h}$. The residual collagenase was removed with PBS. Next, $10 \%$ FBS (Gibco; Thermo Fisher Scientific, Inc.), $100 \mathrm{U} / \mathrm{ml}$ penicillin and $100 \mathrm{mg} / \mathrm{ml}$ streptomycin were added into DMEM/Nutrient Mixture F-12 Ham (Merck-KGaA), and then cultured at $37^{\circ} \mathrm{C}$ with $5 \% \mathrm{CO}_{2}$ after inoculation. The chondrocytes used in this study were passaged twice.

Chondrocytes were obtained and divided into four groups according to different treatments. In the IL- $1 \beta$ group, chondrocytes were treated with $0.5 \mathrm{ng} / \mathrm{ml}$ IL-1 $\beta$ (Sigma-Aldrich; Merck $\mathrm{KGaA}$ ) at $37^{\circ} \mathrm{C}$ for $24 \mathrm{~h}$ to simulate arthritis (18). In the IL-1 $\beta$ + CTS group, chondrocytes were treated with $0.5 \mathrm{ng} / \mathrm{ml}$ IL-1 $\beta$ for $24 \mathrm{~h}$ and $10 \mu \mathrm{M} \mathrm{CTS}$ for $8 \mathrm{~h}$, both at $37^{\circ} \mathrm{C}(18-20)$. In the IL-1 $\beta+$ CTS + microRNA (miRNA/miR)-574-5p mimic and IL-1 $\beta+$ CTS + miR-574-5p mimic + pcDNA-YAF2 groups, chondrocytes were treated with $0.5 \mathrm{ng} / \mathrm{ml} \mathrm{IL-1 \beta}$ for $24 \mathrm{~h}$ and $10 \mu \mathrm{M} \mathrm{CTS}$ for $8 \mathrm{~h}$ at $37^{\circ} \mathrm{C}$. After that, cells were plated into 6-well plates and cultured to $70-80 \%$ confluence, and then transfected with 100 pmol miR-574-5p-mimic (Thermo Fisher Scientific, Inc.), 100 pmol NC mimic and/or $1 \mu \mathrm{g} / \mu \mathrm{l}$ pcDNA-YY1-associated factor 2 (YAF2) at $37^{\circ} \mathrm{C}$ for 48 h using Lipofectamine ${ }^{\circledR} 2000$ (Invitrogen; Thermo Fisher Scientific, Inc.) according to the manufacturer's protocol. After $48 \mathrm{~h}$ of cell transfection, the cells were collected for subsequent experimentation. In the IL-1 $\beta+$ CTS + 5-aza-2'-deoxycytidine (5-aza-CdR; Sigma-Aldrich; Merck KGaA) group, chondrocytes were treated with $0.5 \mathrm{ng} / \mathrm{ml} \mathrm{IL-1} \beta$ for $24 \mathrm{~h}, 10 \mu \mathrm{M}$ CTS for $8 \mathrm{~h}$ and $5 \mu \mathrm{M} 5$-aza-CdR (methylation inhibitor; Sigma-Aldrich; Merck $\mathrm{KGaA}$ ) for $24 \mathrm{~h}$ at $37^{\circ} \mathrm{C}$.

The sequences of the mimics were as follows: Mimic-negative control (NC), 5'-UGGGUUUGUGUGUGU GAGUGUGU-3'; and miR-574-5p mimics, 5'-UGAGUG UGUGUGUGUGAGUGUGU-3'. Mimic-NC and miR-574-5p mimics were synthesized by Shanghai GenePharma Co., Ltd. The full-length sequence of YAF2 cDNA was amplified via PCR and cloned into the pcDNA3.1 vector (Invitrogen; Thermo Fisher Scientific, Inc.) to generate pcDNA-YAF2 constructs by Ke Lei Biotechnology Co., Ltd. An empty vector was used as the corresponding control.

293 T cells were purchased from the Shanghai Cell Bank of the Chinese Academy of Sciences. 293T cells were cultured in high glucose-DMEM containing 10\% (v/v) FBS and 1\% penicillin/streptomycin (Thermo Fisher Scientific, Inc.). The cells were cultured at $37^{\circ} \mathrm{C}$ with $5 \% \mathrm{CO}_{2}$.

$R T$ - $q P C R$. Total RNAs from each sample were extracted using TRIzol ${ }^{\circledR}$ reagent (Vigorous Biotechnology), and then reverse transcribed using a cDNA Synthesis kit (Takara Biotechnology Co., Ltd.) at $37^{\circ} \mathrm{C}$ for $15 \mathrm{~min}$ and $85^{\circ} \mathrm{C}$ for $5 \mathrm{sec}$. A qPCR assay was then used to validate the expression levels of miR-574-5p, miR-468-3p, miR-32-3p and miR-672-5p and YAF2, which was performed on the ABI 7500 Fast Real-Time PCR system 
(Applied Biosystems; Thermo Fisher Scientific, Inc.). The RT-qPCR reaction was conducted in $20 \mu \mathrm{l}$ reaction volumes containing cDNA, primers and SYBR Green Real-time PCR Master mix (Shanghai Yeasen Biotechnology Co., Ltd.). The following thermocycling conditions were used: Initial denaturation at $95^{\circ} \mathrm{C}$ for $5 \mathrm{~min}$; followed by 40 cycles at $95^{\circ} \mathrm{C}$ for $10 \mathrm{sec}, 60^{\circ} \mathrm{C}$ for $30 \mathrm{sec}$ and $72^{\circ} \mathrm{C}$ for $30 \mathrm{sec}$. The $2^{-\Delta \Delta \mathrm{Cq}}$ method was used to access the relative RNA expression levels $(21,22)$. GAPDH and U6 were used as internal references, respectively. The primer sequences are listed in Table I.

Methylation specific PCR (MSPCR). The UCSC database (https://genome.ucsc.edu) was used to predict the promoter sequence of miR-574-5p, and the upstream $2 \mathrm{kB}$ region of the promoter was extracted. MethPrimer (http://www.urogene. org/methprimer), an online analysis site, was used to analyze and predict the $\mathrm{CpG}$ islands of miR-574-5p promoters. The methylation status of miR-574-5p was detected via MSPCR For MSPCR, total genomic DNA was isolated from chondrocytes in the different groups using a Genomic DNA Mini kit (Thermo Fisher Scientific, Inc.) and then $1 \mu \mathrm{g}$ DNA was treated with bisulfate using the EZ DNA Methylation kit (Zymo Research Corp.) according to the manufacturer's instructions. Specific primers in the form of unmethylated or methylated were used: Methylated forward, 5'-TTAATTTAGAATCGG GAAATTTTAC-3' and reverse, 5'-CACTACACCCTAACC TACTACGAA-3; and unmethylated forward, 5'-GAGATC CAAAACTATCAGTCTTGAATGGC-3 and reverse, 5'-GTG TCTGCTCACAGCAGTGAAACCCTAA-3'. The MSPCR products were electrophoresed on $2 \% \mathrm{SYBR}^{\mathrm{TM}}$ safe stained agarose gel.

Western blotting. The expression levels of YAF2, Bcl-2 and Bax were analyzed using western blot analysis. Cells were rinsed in phosphate buffer, and then dissociated using RIPA lysis buffer (EMD Millipore). The concentrations of protein extracts were determined using the Bradford method. An equal amount of protein $(80 \mu \mathrm{g})$ was electrophoresed via $10 \%$ SDS-PAGE, and then transferred to PVDF membranes (EMD Millipore). PVDF membranes were blocked in 5\% non-fat milk at room temperature for $1 \mathrm{~h}$, and then incubated with primary antibodies (anti-YAF2, 1:10,000, cat. no. ab177945; anti-Bax, 1:1,000, cat. no. ab32503; anti-Bcl-2, 1:1,000, cat. no. ab182858; all Abcam) overnight at $4^{\circ} \mathrm{C}$. Next, all membranes were incubated with HRP-labeled secondary antibody $(1: 2,000$; cat. no. ab6721; Abcam) at room temperature for $1 \mathrm{~h}$. Immunoblots were visualized using an ECL kit (Beyotime Institute of Biotechnology) according to the manufacturer's instructions, and then the chemiluminescent signals of protein bands were recorded. GAPDH was used as an internal control.

Von Frey test. The Von Frey test was used to measure the mechanical nociceptive threshold of mice at $0,7,14$ and 21 days (19). Mice were placed on the test bench with a mesh bottom and adapted for $30 \mathrm{~min}$. Then, the hind paw plantar of each mouse was vertically stimulated with calibrated von Frey cilia (0.02-1.4 g; Stoelting Co.) to ensure that the cilia continued to flex for $\geq 2 \mathrm{sec}$ during stimulation. Each mouse was stimulated with cilia of the same intensity for five times, and the behavior of the mice was recorded. The occurrence of related behavioral reactions (foot shrinking, leg swinging, licking, leg lifting, etc.) were recorded as positive reactions, and the minimum stimulus intensity of three positive reactions was recorded as the paw withdrawal threshold (PWT) of the mouse.

$H \& E$ and Safranin $O$ (SO staining). The cartilages of the knee joints of the mice in each group were removed after surgery and rinsed with pre-cooled PBS. After fixation with $4 \%$ paraformaldehyde at room temperature for $48 \mathrm{~h}$, routine paraffin embedding was performed and 5- $\mu \mathrm{m}$ sections were created. A H\&E Staining kit (Beyotime Institute of Biotechnology) was used for H\&E and SO staining. In H\&E staining, the sections were stained with hematoxylin dye solution for $5 \mathrm{~min}$, placed in $1 \%$ hydrochloric acid ethanol differentiation solution for a few sec and rinsed with water. The sections were then stained with eosin solution for $3 \mathrm{~min}$ at room temperature and washed with water. The sections were subsequently dehydrated in gradient alcohol (70, 80, 90 and 100\%) for 5 min and washed with xylene I and II for $5 \mathrm{~min}$. For SO staining, after treatment with $1 \%$ hydrochloric acid ethanol differentiation solution at room temperature for $15 \mathrm{sec}$, sections were soaked in $1 \%$ dilute ammonia for $5 \mathrm{sec}$. After washing with water, sections were stained with Fast Green Stain for 3 min and quickly rinsed with $1 \%$ acetic acid for $10 \mathrm{sec}$. Sections were stained with eosin for $3 \mathrm{~min}$ at room temperature and the following steps were the same as the H\&E staining. Cartilages of knee joints in different groups of mice were observed with a CKX41 phase-contrast microscope (magnifications, $\mathrm{x} 200$ and $\mathrm{x} 400$; Olympus Corporation).

Alcian Blue staining. Chondrocytes at the logarithmic growth stage were inoculated into 6-well plates at a density of $1 \times 10^{5}$ cells/well, and cultured in an incubator of $5 \% \mathrm{CO}_{2}$ at $37^{\circ} \mathrm{C}$. When the cells reached $90 \%$ fusion, they were washed once with PBS, fixed with paraformaldehyde for $10 \mathrm{~min}$ at room temperature, stained with $1 \%$ Alcian blue for $30 \mathrm{~min}$ at $20^{\circ} \mathrm{C}$, and then washed once with $0.1 \% \mathrm{HCl}$ solution. The Alcian Blue Stain kit (Sigma-Aldrich; Merck KGaA) was used for Alcian Blue staining. Cells were visualized using a light microscope (magnification, x1,000; YS110; Nikon Corporation).

TUNEL analysis. TUNEL staining was used to determine the apoptotic status of chondrocytes in different groups, and the apoptotic nuclei were stained brown. TUNEL staining was performed using TUNEL Apoptosis Detection kit IV (FITC+POD; Wuhan Boster Biological Technology, Ltd.) according to the manufacturer's instructions. Briefly, cells were fixed with $4 \%$ paraformaldehyde for $30 \mathrm{~min}$ at room temperature. Before adding TUNEL working solution for $1 \mathrm{~h}$ at $37^{\circ} \mathrm{C}$, DNase I was used to digest the cells for $30 \mathrm{~min}$ to prepare positive cell controls. After adding 3,3'-diaminobenzidine for $15 \mathrm{~min}$ at room temperature, the slides were mounted with neutral balsam mounting media (Sangon Biotech Co., Ltd.) and the apoptotic nuclei showed a yellow-brown color. Apoptotic cells were visualized using a CKX41 phase-contrast microscope (magnifications, $\mathrm{x} 200$ and x400; Olympus Corporation).

Flow cytometry assay. The chondrocytes in the logarithmic growth phase were digested with $0.25 \%$ trypsin (Thermo 
Table I. Primer sequences for reverse transcription-quantitative PCR.

\begin{tabular}{ll}
\hline Gene & \multicolumn{1}{c}{ Primer sequence $\left(5^{\prime} \rightarrow 3^{\prime}\right)$} \\
\hline YY1-associated factor 2 & F: TCGGATGAGGGTTACTGGGACTG \\
miR-574-5p & R: AATCTTGGCCTGGTTTCTTATGG \\
miR-468-3p & F: CGCGTGAGTGTGTGTGTGTGA \\
miR-32-3p & R: AGTGCAGGGTCCGAGGTATT \\
miR-672-5p & F: UAUGACUGAUGUGCGUGUGUCUG \\
GAPDH & R: GACUGAUGUACUGAUAAGAAACUCAGU \\
U6 & F: TTTCTCTATCGATAGGTACCGGCAGTTACCATTTCACAC \\
& R: CACGCCGAATCAACATCAGTCTGATAA \\
& F: UGAGGUUGGUGUACUGUGUGUGA \\
& R: ACACACAGUCGCCAUCUUCGA \\
& F: TGCCCCCATGTTCGTCA \\
& R: TTGGCCAGGGGTGCTAAG \\
& F: CTCGCTTCGGCAGCACA \\
& R: AACGCTTCACGAATTTGCGT
\end{tabular}

F, forward; R, reverse; $\mathrm{miR}$, microRNA.

Fisher Scientific, Inc.) and inoculated into 6-well cell culture plates at a density of $2 \times 10^{6}$ cells/well. The Annexin V FITC APOPTOSIS Detection kit I 100TST (BD Pharmingen; BD Biosciences) was used according to the manufacturer's instructions. Briefly, $5 \mu \mathrm{l}$ Annexin V-FITC and $10 \mu \mathrm{l}$ propidium iodide (PI) was added, and cells were incubated at room temperature for 15 min in dark. A CytoFLEX flow cytometer (Beckman Coulter, Inc.) was used to detect cell fluorescence immediately after staining with PI solution at room temperature. FlowJo Software 10 (FlowJo LLC) was used for data analysis. Early and late apoptotic cells were counted to determine the levels of cardiomyocyte apoptosis.

Dual-luciferase assay. First, the binding sites of miR-574-5p and YAF2 were predicted via the miRDB database (http://www.mirdb.org), and the binding sites were amplified by PCR. Then, the binding sites were inserted into the pmirGLO reporter vector (Promega Corporation) to construct the YAF2 wild-type (WT) plasmid (Luc-WT-YAF2). The YAF2 mutant (MUT) plasmid (Luc-MUT-YAF2) was constructed by mutating nucleotides using gene mutation technology. After cells were plated into 6-well plates and cultured to $70-80 \%$ confluence, they were co-transfected with $0.25 \mu \mathrm{g}$ Luc-WT-YAF2, $0.25 \mu \mathrm{g}$ Luc-MUT-YAF2 and $0.25 \mu \mathrm{g}$ miR-574-5P-mimic or $0.25 \mu \mathrm{g}$ mimic-NC into $293 \mathrm{~T}$ cells at $37^{\circ} \mathrm{C}$ for $24 \mathrm{~h}$ using Lipofectamine ${ }^{\circledR} 2000$ (Invitrogen; Thermo Fisher Scientific, Inc.). The Luciferase Assay kit (Invitrogen; Thermo Fisher Scientific, Inc.) was used to detect the luciferase activity of each group. Normalized relative luciferase activity=Firefly luciferase activity/Renilla luciferase activity.

Cell proliferation detection. Cell proliferation detection was conducted according to the Cell Counting Kit-8 (CCK-8) instructions (MedChemExpress). Chondrocytes at the logarithmic growth stage were inoculated into 96 -well plates $\left(1 \times 10^{5}\right.$ cells/well $)$ and incubated in an incubator $\left(37^{\circ} \mathrm{C} ; 5 \% \mathrm{CO}_{2}\right)$ for $48 \mathrm{~h}$. Next, $100 \mu \mathrm{l} \mathrm{CCK-8} \mathrm{solution} \mathrm{(10 \%} \mathrm{concentration)}$ was added for further culture for $1 \mathrm{~h}$ at $37^{\circ} \mathrm{C}$. The absorbance value (optical density value) at $450 \mathrm{~nm}$ was determined using a microplate reader. All the experiments were repeated three times.

Statistical analysis. SPSS 20.0 software (IBM Corp.) was used for statistical analysis of data collected from $\geq 3$ independent experiments. The measurement data are presented as the mean \pm SD. An unpaired Student's t-test was used to analyze the difference between the two groups. One-way ANOVA and Tukey's post hoc test were used to analyze the data between multiple groups. $\mathrm{P}<0.05$ was considered to indicate a statistically significant difference.

\section{Results}

Expression levels of miR-574-5p, YAF2, Bax and Bcl-2 in the mouse model of $O A$. Differential expression analysis based on the microarray data set GSE93008 (Sham vs. OA, n=3 vs. n=3) of OA mice was performed. Taking the adjusted $\mathrm{P}$-value $<0.05$ and $\mid \log \mathrm{FCl} \geq 1$ as screening conditions, four conservative miRNAs, namely miR-574-5p, miR-468-3p, miR-32-3p and miR-672-5p, were identified (data not shown). RT-qPCR was used to detect the expression levels of four conserved miRNAs in the Sham group and OA group, and the results demonstrated that miR-574-5p had the most significant changes, indicating that miR-574-5p may be an important regulatory factor affecting OA (Fig. 1A).

Yang et al (23) reported that YAF2 has a protective effect on chondrocytes by inhibiting cell apoptosis. Therefore, RT-qPCR was performed to detect the expression levels of miR-574-5p and YAF2 in the Sham group, OA group and $\mathrm{OA}+\mathrm{CTS}$ group. The results demonstrated that the expression level of miR-574-5P was the highest in the OA group, and was downregulated in the OA + CTS group (Fig. 1B). However, 


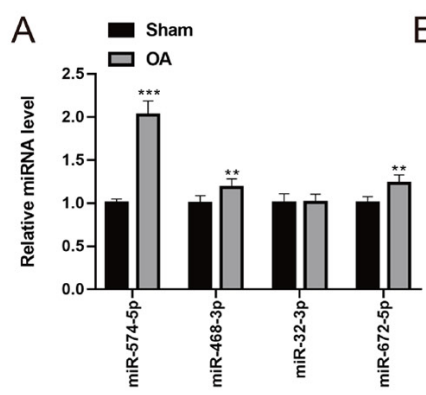

E

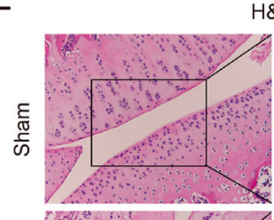

H\&E

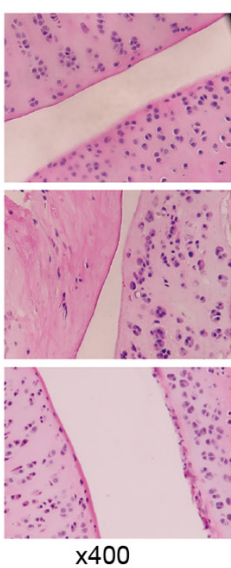

B

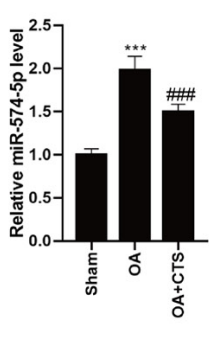

C

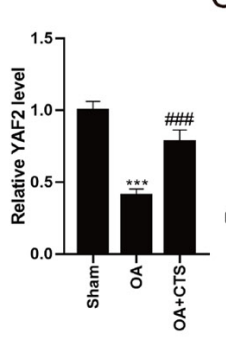

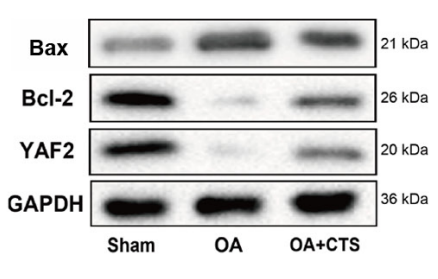

D - Sham

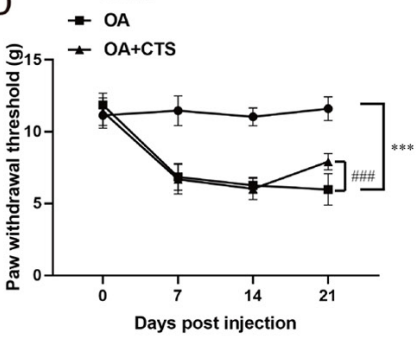

$\mathrm{F}$

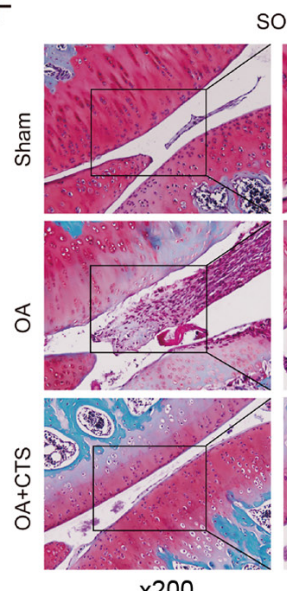

so

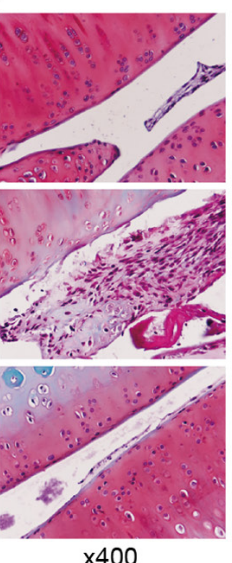

G
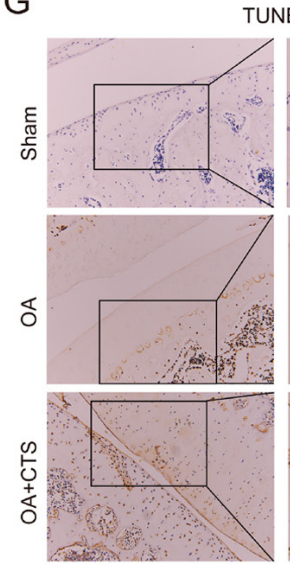

$\times 200$
TUNEL

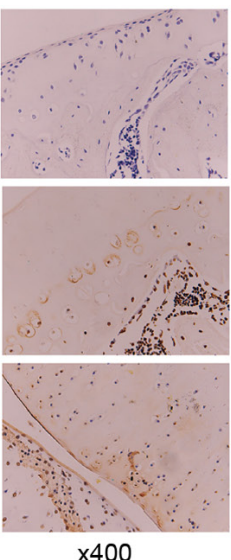

Figure 1. Expression levels of miR-574-5p, YAF2, Bax and Bcl-2 in the mouse model of OA. (A) Differential expression analysis based on the microarray data set GSE93008 (Sham vs. OA, n=3 vs. n=3) of OA mice was performed. RT-qPCR was used to detect the expression levels of four conserved miRNAs in the Sham group and OA group. (B) RT-qPCR was performed to detect the expression levels of miR-574-5p and YAF2 in the Sham group, OA group and OA + CTS group. (C) Western blot analysis was used to detect the protein expression levels of YAF2, Bax and Bcl-2 in OA mice. (D) Von Frey test was used to measure mechanical nociceptive threshold of mice at 0,7,14 and 21 days. (E) H\&E and (F) SO staining were performed to reveal the morphology of mouse cartilage between Sham group, OA group and OA + CTS group. (G) Chondrocyte apoptosis was detected using a TUNEL assay. Magnifications, x200 and x400. ${ }^{* *} \mathrm{P}<0.01,{ }^{* * *} \mathrm{P}<0.001$ vs. Sham group; ${ }^{\# \# \#} \mathrm{P}<0.001$ vs. OA group. OA, osteoarthritis; CTS, Cryptotanshinone; miRNA/miR, microRNA; YAF2, YY1-associated factor 2; SO, Safranin O; RT-qPCR, reverse transcription-quantitative PCR.

compared with the Sham group, YAF2 expression in the OA group was significantly decreased, while YAF2 expression in the OA + CTS group was increased (Fig. 1B and C). In addition, the expression levels of Bcl-2 and Bax, which inhibit and promote cell apoptosis (24), respectively, were measured. The western blotting results indicated that the protein expression level of Bcl-2 in OA mice was markedly downregulated, but was increased after the gavage of CTS suspension, while the protein expression level of Bax exhibited the opposite effects (Fig. 1C).

Effect of CTS on chondrocyte apoptosis in vivo. To investigate the effect of OA on pain sensation in mice, the present study compared the differences in PWT between Sham group, OA group and OA + CTS group. The bilateral PWT in the OA group was detected using a Von Frey test, and the results demonstrated that there was no significant change between the Sham group and the OA-contralateral group at day 21, while the PWT in the OA-ipsilateral group was markedly decreased compared with the Sham and OA-contralateral groups (Fig. S1). Thus, this study only compared the unilateral PWT between the Sham group, OA group and OA + CTS group. As presented in Fig. 1D, when compared with the Sham group, the PWT of mice in the OA group at day 21 was significantly reduced, and the PWT was significantly increased at day 21 in the OA + CTS group compared with the OA group, indicating that CTS could relieve the pain of mice with OA.

Next, HE and SO staining were conducted to reveal the morphology of mouse cartilage between the Sham group, OA group and OA + CTS group. In the Sham group, it was found that the cartilage remained intact and the chondrocytes (blue) were ordered neatly, while the cartilage was thinner and the number of chondrocytes was decreased in the OA group (Fig. 1E). After the addition of CTS, the apoptosis of chondrocytes was alleviated (Fig. 1E). SO staining of the cytoplasmic matrix in the Sham group was normal, while the degree of the cytoplasmic matrix in the OA group was increased and was recovered in the OA + CTS group (Fig. 1F). In addition, TUNEL staining results identified that there was no positive nuclear staining in the Sham group, and that the number of apoptotic cells in the OA group was markedly increased, while the number of apoptotic cells was significantly decreased after adding CTS (Fig. 1G).

Expression levels of miR-574-5p, YAF2, Bax and Bcl-2 in chondrocytes. Next, it was verified whether the expression levels of miR-574-5p, YAF2, Bax and Bcl-2 in chondrocytes were consistent with those in the mouse model of OA. The successful differentiation of chondrocytes was first determined using Alcian Blue staining (Fig. 2A), and then the chondrocytes 
A

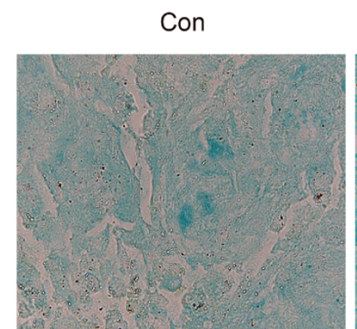

$\mathrm{x} 1000$
Chondrocytes

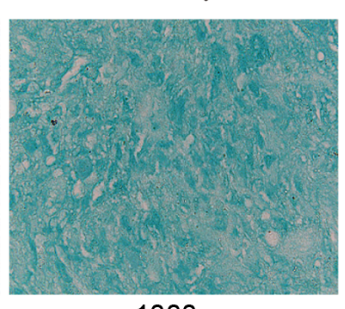

$x 1000$
B

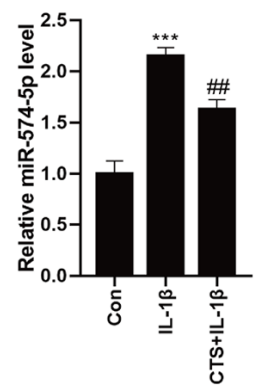

C

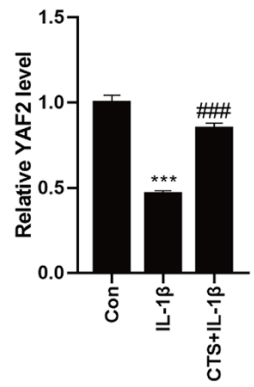

E
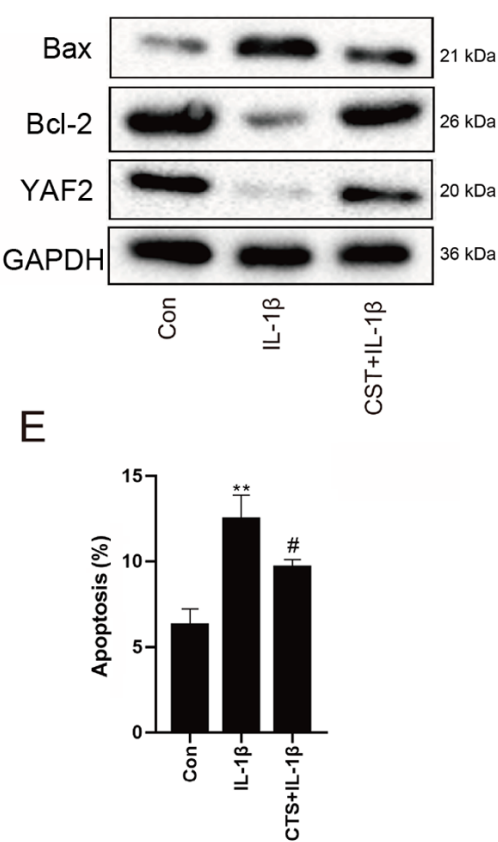

Figure 2. Expression levels of miR-574-5p, YAF2, Bax and Bcl-2 in chondrocytes. Chondrocytes were divided into Con group, IL-1 $\beta$ group and IL-1 $\beta+$ CTS group after different treatments. (A) Alcian Blue staining was used to detect the differentiation of chondrocytes. Magnification, $\mathrm{x} 1,000$ (B) Differential expression levels of miR-574-5p and YAF2 in chondrocytes were detected via reverse transcription-quantitative PCR. (C) Western blot analysis was used to detect the protein expression levels of YAF2, Bax and Bcl-2 in chondrocytes. (D) Apoptotic rate of chondrocytes was detected via flow cytometry, and (E) the results were quantified. ${ }^{* *} \mathrm{P}<0.01,{ }^{* * *} \mathrm{P}<0.001$ vs. control group; ${ }^{\#} \mathrm{P}<0.05,{ }^{\# \#} \mathrm{P}<0.01,{ }^{\# \#} \mathrm{P}<0.001$ vs. IL-1 $\beta$ group. Con, control; CTS, Cryptotanshinone; miR, microRNA; YAF2, YY1-associated factor 2.

were divided into control group, IL-1 $\beta$ group and IL-1 $1 \beta+$ CTS group after different treatments. The differential expression levels of miR-574-5p and YAF2 in chondrocytes were detected via RT-qPCR, and the results indicated that the expression level of miR-574-5p in the IL-1 $\beta$ group was increased, but was decreased in the IL-1 $\beta$ + CTS group (Fig. 2B). By contrast, YAF2 mRNA and protein expression levels were both decreased in the IL-1 $\beta$ group compared with control group, and were increased after the addition of CTS (Fig. 2B and C).

Western blot analysis revealed that, compared with control group, the protein level of Bax in the IL- $1 \beta$ group was markedly increased, while the expression of Bcl-2 was decreased. After adding CTS, the protein expression level of Bax was downregulated, while the expression level of $\mathrm{Bcl}-2$ was upregulated (Fig. 2C).

As shown in Fig. 2D and E, the apoptotic rate of chondrocytes in the IL- $1 \beta$ group detected by flow cytometry was higher compared with that in the control group, while the apoptotic rate in the IL- $1 \beta+$ CTS group was decreased. All these findings suggested that the expression levels of miR-574-5p and the pro-apoptotic gene Bax were upregulated, while the expression levels of YAF2 and the anti-apoptotic gene Bcl-2 were downregulated in arthritic chondrocytes, indicating that CTS could alleviate the apoptosis of chondrocytes.

CTS affects chondrocyte apoptosis by regulating the expression of miR-574-5p and then interfering with YAF2. Since the biogenic analysis found that miR-574-5p may target binding to YAF2 (Fig. 3A), a dual-luciferase reporter assay was performed to determine whether miR-574-5p could interact with the YAF2 promoter. The 293T cells were co-transfected with the YAF2 promoter-luciferase reporter plasmid and mimic-NC or miR-574-5p mimic. As presented in Fig. 3B, in the Luc-WT-YAF2 group, the luciferase activity of the miR-574-5p mimic was significantly decreased compared with that in 293T cells transfected with mimic-NC, indicating that miR-574-5p had a regulating effect on YAF2. The mRNA expression levels of YAF2 were validated in Fig. S2.

After treatment with IL-1 $\beta$ and CTS, chondrocytes were transfected with miR-574-5p-mimics and then co-transfected with pcDNA-YAF2. The mRNA expression levels of miR-574-5p and YAF2 after cell transfection with miR-574-5p mimic and pcDNA-YAF2 were detected via RT-qPCR (Fig. S3). The expression level of miR-574-5p in arthritic chondrocytes with addition of IL-1 $\beta$ was increased, and then decreased after the addition of CTS (Fig. 3C), indicating that CTS could reduce the expression of miR-574-5p in arthritic chondrocytes. After chondrocytes treated with IL-1 $\beta$ and CTS, and then transfected with miR-574-5p-mimics, miR-574-5p expression was found to be upregulated (Fig. 3C). In addition, there was no significant change in the expression level of miR-574-5p after overexpressing YAF2 (Fig. 3C). Opposite results were found for the mRNA and protein expression levels of YAF2, indicating that overexpression of miR-574-5p could decrease the expression of YAF2 (Fig. 3D and E). Transfection with pcDNA-YAF2 reversed the effect of miR-574-5p mimic, as YAF2 expression was significantly increased (Fig. 3D and E).

Bax expression was upregulated in arthritic chondrocytes and was downregulated after the addition of CTS, which was 
A

\begin{tabular}{|c|c|c|c|}
\hline \multicolumn{2}{|c|}{ ENSMUSG000C0022634 (Yaf2) } & mmu-miR-574-5p & 0.893616802291285 \\
\hline Region & Binding Type & Transcript position & Score \\
\hline UTR3 & $6 \mathrm{mer}$ & $41-58$ & 0.00452652429512702 \\
\hline Binding area: & (Iranscr & 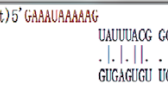 & $\begin{array}{l}3^{3} \\
\text { cadoca } \\
\text { IIIIIII } \\
\text { GUGAGL }\end{array}$ \\
\hline & (miRNA) & $3^{\prime}$ & U 5 ' \\
\hline
\end{tabular}

D
B

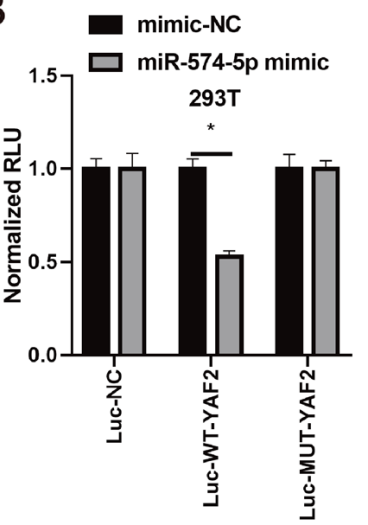

C

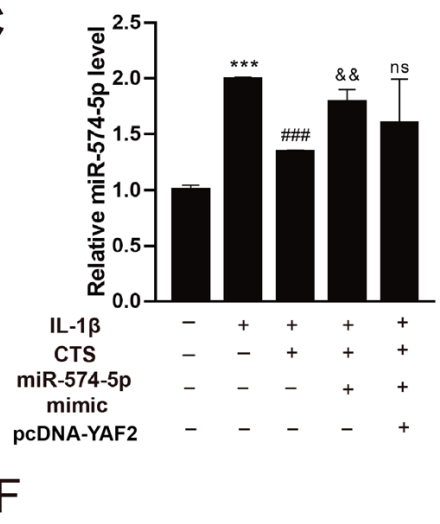

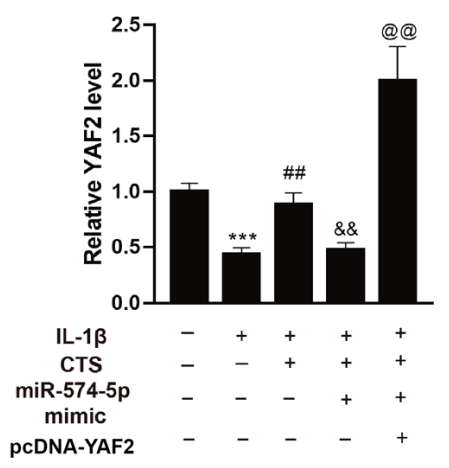

G

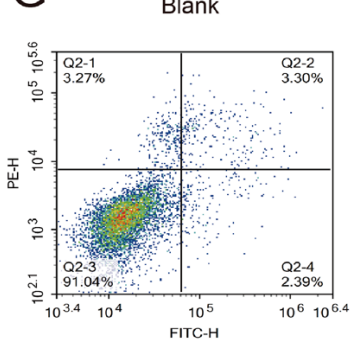

E

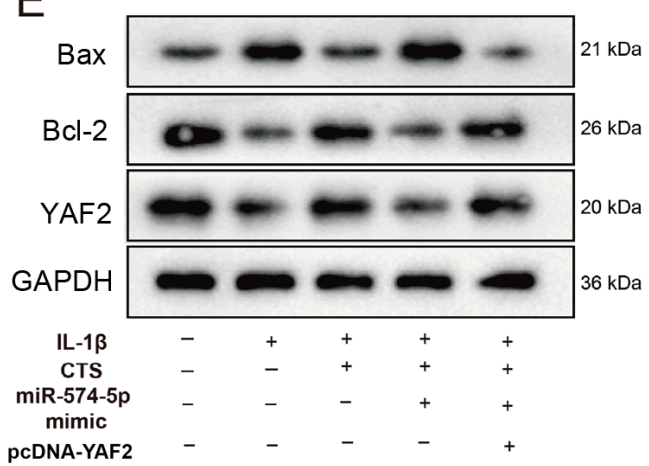

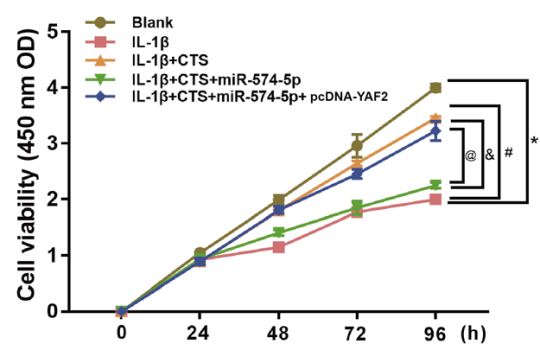

IL-1 $\beta$

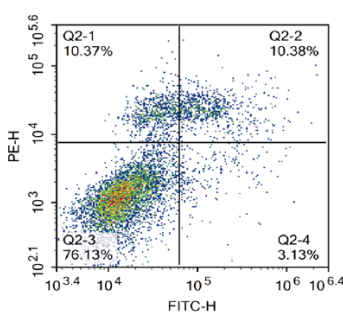

IL-1 $1 \beta+C T S$

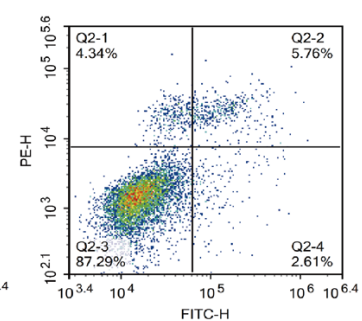

IL- $1 \beta+C T S$ + miR-574-5p mimic

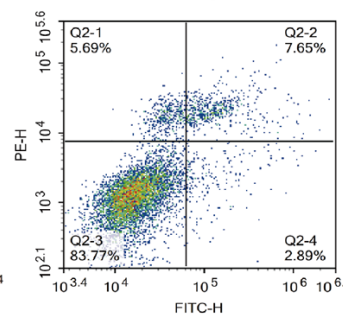

IL-1 $\beta+C T S+m i R-574-5 p$ mimic + pcDNA-YAF2

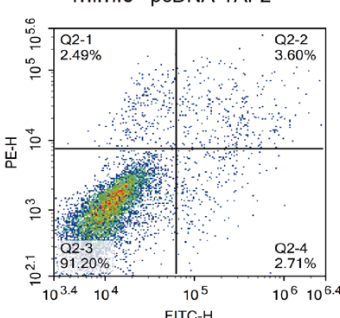

Figure 3. CTS affects chondrocyte apoptosis by regulating the expression of miR-574-5p and then interfering with YAF2. (A) Biogenic analysis and (B) dual-luciferase reporter assay was preformed to verify the interaction between miR-574-5p and YAF2. Chondrocytes were divided into Blank group, IL-1 $\beta$ group, IL-1 $\beta$ + CTS group, IL-1 $\beta$ + CTS + miR-574-5p-mimic group and IL-1 $\beta+$ CTS + miR-574-5p-mimic + pcDNA-YAF2 group. * P 0.05 . Differential expression levels of (C) miR-574-5p and (D) YAF2 in chondrocytes were detected via reverse transcription-quantitative PCR. (E) Western blot analysis was used to detect the protein expression levels of YAF2, Bax and Bcl-2 in chondrocytes. Cell proliferation and apoptosis of chondrocytes were detected using a

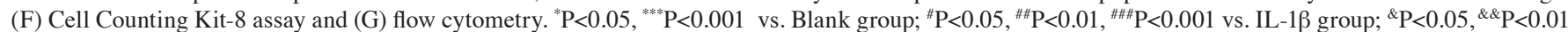
vs. IL-1 $\beta+$ CTS group; ${ }^{\circledR} \mathrm{P}<0.05,{ }^{\circledR} \mathrm{P}<0.001$ vs. IL-1 $\beta+\mathrm{CTS}+$ miR-574-5p-mimic group. CTS, Cryptotanshinone; miR, microRNA; YAF2, YY1-associated factor 2; OD, optical density; Luc, luciferase; NC, negative control; RLU, relative luciferase activity; WT, wild-type; MUT, mutant.

upregulated after IL-1 $\beta+$ CTS-treated cells were transfected with miR-574-5p-mimic. Moreover, after transfection with pcDNA-YAF2, the expression of Bax was decreased (Fig. 3E). By contrast, the protein expression level of Bcl-2 showed an opposite trend, indicating that miR-574-5p could increase Bax expression and decrease Bcl-2 expression (Fig. 3E).

Next, a CCK-8 assay was performed to detect the effects of CTS, miR-574-5p and YAF2 on chondrocytes proliferation. The results demonstrated that the proliferative ability of chondrocytes in the IL-1 $\beta$ group was significantly decreased, but this was restored after the addition of CTS. However, the proliferative ability of chondrocytes after transfection with miR-574-5p-mimic was decreased to a level similar to that of the IL-1 $\beta$ group (Fig. 3F). Furthermore, transfection with pcDNA-YAF2 could reverse the effect of miR-574-5p, and the proliferative ability was significantly increased.
Flow cytometry results identified that the apoptotic rate of OA chondrocytes was markedly increased compared with the Blank group, while the apoptotic rate in the IL-1 $\beta+$ CTS group was decreased and was increased after transfection with miR-574-5p-mimic (Fig. 3G). Moreover, the addition of YAF2 decreased the apoptotic rate of chondrocytes. These results suggested that CTS could affect chondrocyte proliferation and apoptosis by regulating the expression of miR-574-5p and then interfering with YAF2.

$C T S$ regulates the methylation of the miR-574-5p promoter. Since it was found that the CTS/miR-574-5p/YAF2 signaling pathway could affect the proliferation and apoptosis of arthritic chondrocytes, this study further aimed to investigate how CTS regulates the expression of miR-574-5p and thus affects YAF2 expression. It has been reported that CTS can upregulate the 

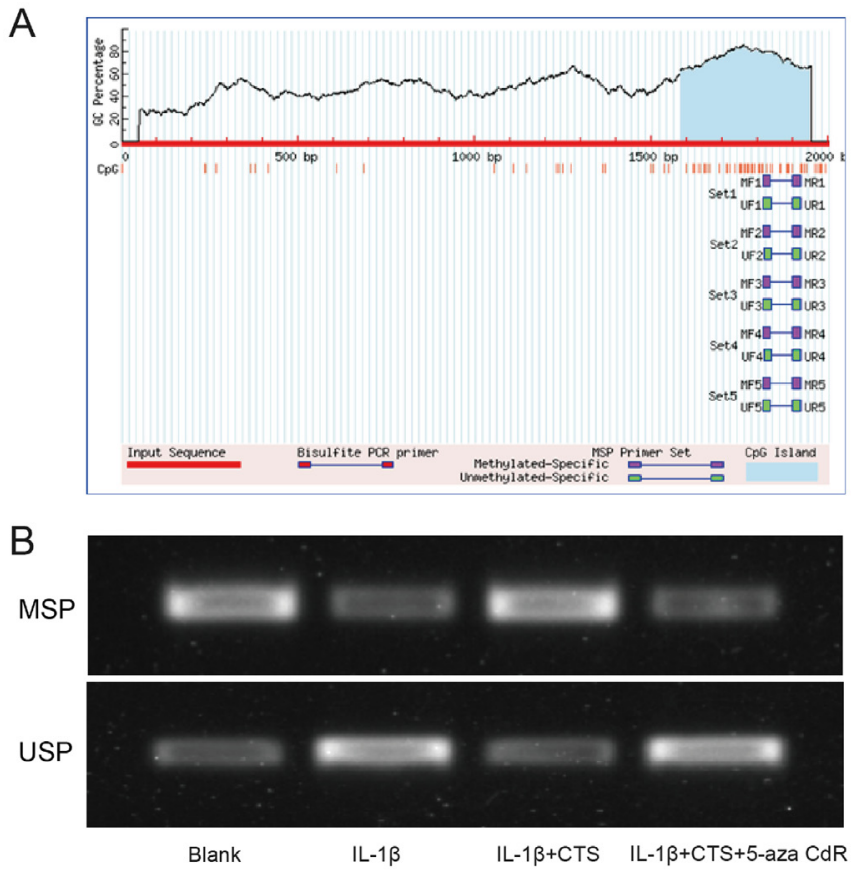

Figure 4. CTS regulates the methylation of the miR-574-5p promoter. (A) The upstream $2 \mathrm{~kb}$ promoter region of miR-574-5p was detected via biogenic analysis. (B) Methylation levels of miR-574-5p promoter in the Blank group, IL- $1 \beta$ group, IL-1 $\beta+$ CTS group and IL-1 $\beta+$ CTS + 5-aza-CdR group were measured using MSPCR. MSPCR, Methylation specific PCR; MSP, methylated; USP, unmethylated; CTS, Cryptotanshinone; miR, microRNA; 5-aza-CdR, 5-aza-2'-deoxycytidine.

methylation levels of some molecular promoters in prostate cancer (25). Therefore, the $2 \mathrm{~kb}$ region upstream of miR-574-5p was examined, and the presence of $\mathrm{CpG}$ island was found (Fig. 4A), indicating that CTS may regulate miR-574-5p expression by affecting its methylation. The methylation levels of the miR-574-5p promoter in the Blank group, IL-1 $\beta$ group, IL- $1 \beta+$ CTS group and IL- $1 \beta+$ CTS + 5-aza-CdR group were measured via MSPCR. The methylation degree of the miR-574-5p promoter was reduced in arthritic chondrocytes, but was restored after the addition of CTS. After the addition of the methylation inhibitor 5-aza-CdR, the methylation degree of the miR-574-5p promoter was reduced, indicating that CTS could regulate the methylation of the miR-574-5p promoter (Fig. 4B).

\section{Discussion}

Non-coding RNA (ncRNA) is a type of RNA that does not encode proteins. According to the length of ncRNA fragments, they can be divided into short ncRNA, such as small interfering RNA, piwi-interacting RNA, miRNA and long non-coding RNA (lncRNA) (26). ncRNAs are involved in almost every step of the life cycle, from gene transcription to mRNA splicing to RNA decay and translation (27).

miRNAs are a class of small molecular ncRNAs with a length of 20-23 nucleotides. Previous studies have reported that miRNAs, as an important regulatory factor, are often observed in various diseases, and $\sim 1 / 2$ of the mRNAs are regulated by miRNAs $(28,29)$. Moreover, studies have confirmed that miRNAs and IncRNAs are involved in regulating $\mathrm{OA}$ progression by regulating chondrocyte activity and function (27-29). In order to identify key miRNAs that can affect OA, the present study established an OA mouse model. The C57BL/6 mice used in this study are easy to feed and are often used as experimental animal models of physiology and pathology, such as obesity, type 2 diabetes, atherosclerosis and osteoporosis, and also used as an OA model $(15,30)$. Next, the current study conducted bioinformation analysis on the OA mouse data set and found four conserved miRNAs, among which the expression level of miR-574-5p was the highest in the knee joint of mice with OA. Based on the protective effect of CTS on chondrocytes and the differential expression of miR-574-5p, it was suggested that CTS may promote chondrocyte proliferation and inhibit its apoptosis by regulating the expression of miR-574-5p, thus alleviating OA. However, the specific signal pathway remains unknown.

YAF2, a zinc finger protein, was first identified due to its interaction with the transcription factor Yin Yang 1, which has an effect on the proliferation of tumor cells (31). In genotoxic stress reactions, YAF2 can positively regulate the function of programmed cell death 5 in the human lung adenocarcinoma cell line A549 and human melanoma cell line HCT116 (32). Other studies have shown that the interaction between YAF2 and polycomb group protein can inhibit the apoptosis induced by caspase 8 during the early embryonic development of zebrafish (33). Based on the protective effect of YAF2 on chondrocytes by regulating apoptosis, the present study found that expression levels of YAF2 and the anti-apoptotic gene Bcl-2 were significantly decreased in mice with OA, while the expression levels of miR-574-5p and the pro-apoptotic gene Bax showed opposite changes. The in vitro experiments demonstrated that the expression levels of miR-574-5p, YAF2, Bax and Bcl-2 in chondrocytes were consistent with the in vivo findings. Furthermore, the addition of CTS could alter the effect, which verified that CTS could alleviate OA.

Next, the present study aimed to determine how CTS regulates the expression levels of miR-574-5P and YAF2, to ultimately affect chondrocyte apoptosis. Using biogenic analysis and verification experiments, it was identified that miR-574-5p could interact with the YAF2 promoter, and exerted a regulating effect on YAF2. The miR-574-5P/YAF2 pathway has been confirmed, but it is necessary to further study how CTS regulates the expression of miR-574-5p. As a potential active ingredient in the treatment of prostate cancer, CTS could regulate the methylation of the key factor histone $\mathrm{H} 3$ lysine 9 (25). After the analysis and prediction, the present study further found that the methylation degree of the miR-574-5p promoter was increased by the effect of CTS, and was reduced after the addition of the methylation inhibitor.

In conclusion, the present study provided novel targets for the treatment of OA with CTS and validated that miR-574-5p is an important regulatory factor affecting OA. Furthermore, the present study investigated the mechanism via which CTS affects chondrocyte apoptosis by regulating the methylation of miR-574-5P, thus interfering with YAF2.

\section{Acknowledgements}

Not applicable. 


\section{Funding}

This study was supported by Special project of Scientific Research on Traditional Chinese Medicine of Henan Province (grant no. 2018ZY2019).

\section{Availability of data and materials}

The datasets used and/or analyzed during the current study are available from the corresponding author on reasonable request.

\section{Authors' contributions}

SY and MG designed the study. SY and XS wrote the manuscript. MG supervised the study. JT and JW analyzed the data. SY and JW prepared the figures. The experiments were performed by XS, JT and JW. SY and MG confirm the authenticity of all the raw data. All authors read and approved the final manuscript.

\section{Ethics approval and consent to participate}

All animal experiments were approved by the Animal Ethics Committee of Orthopedic-Traumatological Hospital of Henan Province (Henan Provincial Orthopedic Hospital) (approval no. KY201902046).

\section{Patient consent for publication}

Not applicable.

\section{Competing interests}

The authors declare that they have no competing interests.

\section{References}

1. Loeser RF, Collins JA and Diekman BO: Ageing and the pathogenesis of osteoarthritis. Nat Rev Rheumatol 12 : 412-420, 2016

2. Keen HI, Hensor EMA, Wakefield RJ, Mease PJ, Bingham CO III and Conaghan PG: Ultrasound assessment of response to intra-articular therapy in osteoarthritis of the knee. Rheumatology (Oxford) 54: 1385-1391, 2015.

3. Bellot G, Garcia-Medina R, Gounon P, Chiche J, Roux D, Pouysségur J and Mazure NM: Hypoxia-induced autophagy is mediated through hypoxia-inducible factor induction of BNIP3 and BNIP3L via their BH3 domains. Mol Cell Biol 29: 2570-2581, 2009.

4. Cleveland RJ, Alvarez C, Schwartz TA, Losina E, Renner JB, Jordan JM and Callahan LF: The impact of painful knee osteoarthritis on mortality: A community-based cohort study with over 24 years of follow-up. Osteoarthritis Cartilage 27: 593-602, 2019.

5. Nho SJ, Kymes SM, Callaghan JJ and Felson DT: The burden of hip osteoarthritis in the United States: Epidemiologic and economic considerations. J Am Acad Orthop Surg 21 (Suppl 1): S1-S6, 2013

6. Hosseinzadeh A, Kamrava SK, Joghataei MT, Darabi R, Shakeri-Zadeh A, Shahriari M, Reiter RJ, Ghaznavi H and Mehrzadi S: Apoptosis signaling pathways in osteoarthritis and possible protective role of melatonin. J Pineal Res 61: 411-425, 2016.

7. Vuppalapati KK, Bouderlique T, Newton PT, Kaminskyy VO, Wehtje H, Ohlsson C, Zhivotovsky B and Chagin AS: Targeted deletion of autophagy genes Atg5 or Atg7 in the chondrocytes promotes caspase-dependent cell death and leads to mild growth retardation. J Bone Miner Res 30: 2249-2261, 2015.
8. Varela-Eirin M, Loureiro J, Fonseca E, Corrochano S, Caeiro JR, Collado M and Mayan MD: Cartilage regeneration and ageing: Targeting cellular plasticity in osteoarthritis. Ageing Res Rev 42: 56-71, 2018

9. Charlier E, Relic B, Deroyer C, Malaise O, Neuville S, Collée J, Malaise MG and De Seny D: Insights on molecular mechanisms of chondrocytes death in osteoarthritis. Int J Mol Sci 17: 2146 , 2016.

10. Guo M, Guo J, Cui H, Guo Y and Guo Y: Effects of Zhuanggu huoxue tang on gucuronic acid and water contents in cartilage of rabbits with osteoarthritis. Chin J Tradit Med Sci Technol 23: 538-539, 2016.

11. Tao J, Wang S, Chen Y, Zheng H, Jiang M and Yuan B: Review on the research in network pharmacology of traditional Chinese medicine compound. China J Tradit Chin Med Pharm 34: 3903-3907, 2019.

12. Liu L, Liu HP, Xie JR, Yang LY, Wang MY, Peng T, Liu PA and Zhang GM: Association analysis on effective components in Zhuanggu Zhitong prescription. Chin Tradit Herbal Drugs 49: 1451-1460, 2018.

13. Ashrafizadeh M, Zarrabi A, Orouei S, Saberifar S, Salami S, Hushmandi K and Najafi M: Recent advances and future directions in anti-tumor activity of cryptotanshinone: A mechanistic review. Phytother Res 35: 155-179, 2021.

14. Wu YH, Wu YR, Li B and Yan ZY: Cryptotanshinone: A review of its pharmacology activities and molecular mechanisms. Fitoterapia 145: 104633, 2020.

15. Feng Z, Zheng W, Li X, Lin J, Xie C, Li H, Cheng L, Wu A and $\mathrm{Ni}$ W: Cryptotanshinone protects against IL- $1 \beta$-induced inflammation in human osteoarthritis chondrocytes and ameliorates the progression of osteoarthritis in mice. Int Immunopharmacol 50: 161-167, 2017

16. Kung LHW, Ravi V, Rowley L, Angelucci C, Fosang AJ, Bell KM, Little CB and Bateman JF: Cartilage MicroRNA dysregulation during the onset and progression of mouse osteoarthritis is independent of aggrecanolysis and overlaps with candidates from end-stage human disease. Arthritis Rheumatol 70: 383-395, 2018.

17. Ouyang J, Jiang H, Fang H, Cui W and Cai D: Isoimperatorin ameliorates osteoarthritis by downregulating the mammalian target of rapamycin $\mathrm{C} 1$ signaling pathway. Mol Med Rep 16: 9636-9644, 2017

18. Pirozzi C, Francisco V, Guida FD, Gómez R, Lago F, Pino J, Meli R and Gualillo O: Butyrate modulates inflammation in chondrocytes via GPR43 receptor. Cell Physiol Biochem 51: 228-243, 2018

19. Na HS, Park JS, Cho KH, Kwon JY, Choi J, Jhun J, Kim SJ, Park SH and Cho ML: Interleukin-1-interleukin-17 signaling axis induces cartilage destruction and promotes experimental osteoarthritis. Front Immunol 11: 730, 2020.

20. Ji Q, Qi D, Xu X, Xu Y, Goodman SB, Kang L, Song Q, Fan Z, Maloney WJ and Wang Y: Cryptotanshinone protects cartilage against developing osteoarthritis through the miR-106a-5p/GLIS3 axis. Mol Ther Nucleic Acids 11: 170-179, 2018.

21. Livak KJ and Schmittgen TD: Analysis of relative gene expression data using real-time quantitative PCR and the 2(-Delta Delta C(T)) method. Methods 25: 402-408, 2001.

22. Barra GB, Santa Rita TH, Almeida ALSC, Jácomo RH and Nery LFA: Serum has higher proportion of janus kinase 2 V617F mutation compared to paired EDTA-whole blood sample: A model for somatic mutation quantification using qPCR and the 2- $\Delta \Delta \mathrm{Cq}$ method. Diagnostics (Basel) 10: 153, 2020.

23. Yang Q, Li X, Zhou Y, Fu W, Wang J and Wei Q: A LINC00341-mediated regulatory pathway supports chondrocyte survival and may prevent osteoarthritis progression. J Cell Biochem 120: 10812-10820, 2019.

24. Knudson CM and Korsmeyer SJ: Bcl-2 and Bax function independently to regulate cell death. Nat Genet 16: 358-363, 1997.

25. Wu CY, Hsieh CY, Huang KE, Chang C and Kang HY: Cryptotanshinone down-regulates androgen receptor signaling by modulating lysine-specific demethylase 1 function. Int J Cancer 131: 1423-1434, 2012

26. Dong X, Dong X, Gao F, Liu N, Liang T, Zhang F, Fu X, Pu L and Chen J: Non-coding RNAs in cardiomyocyte proliferation and cardiac regeneration: Dissecting their therapeutic values. J Cell Mol Med 25: 2315-2332, 2021.

27. Razmara E, Bitaraf A, Yousefi H, Nguyen TH, Garshasbi M, Cho WC and Babashah S: Non-coding RNAs in cartilage development: An updated review. Int J Mol Sci 20: 4475, 2019. 
28. Zhang Y, Wang G, Ma L, Wang C, Wang L, Guo Y and Zhao X: miR-137 suppresses cell growth and extracellular matrixdegradation through regulating ADAMTS-5 in chondrocytes. Am J Transl Res 11: 7027-7034, 2019.

29. Swingler TE, Niu L, Smith P, Paddy P, Le L, Barter MJ, Young DA and Clark IM: The function of microRNAs in cartilage and osteoarthritis. Clin Exp Rheumatol 37 (Suppl 120): S40-S47, 2019

30. Monteagudo S, Cornelis FMF, Aznar-Lopez C, Yibmantasiri P, Guns LA, Carmeliet P, Cailotto F and Lories RJ: DOT1L safeguards cartilage homeostasis and protects against osteoarthritis. Nat Commun 8: 15889, 2017.

31. Kalenik JL, Chen D, Bradley ME, Chen SJ and Lee TC: Yeast two-hybrid cloning of a novel zinc finger protein that interacts with the multifunctional transcription factor YY1. Nucleic Acids Res 25: 843-849, 1997.
32. Park SY, Choi HK, Jo SH, Seo J, Han EJ, Choi KC, Jeong JW, Choi Y and Yoon HG: YAF2 promotes TP53-mediated genotoxic stress response via stabilization of PDCD5. Biochim Biophys Acta 1853: 1060-1072, 2015.

33. Stanton SE, McReynolds LJ, Evans T and Schreiber-Agus N: Yaf2 inhibits caspase 8-mediated apoptosis and regulates cell survival during zebrafish embryogenesis. J Biol Chem 281: 28782-28793, 2006

This work is licensed under a Creative Commons Attribution-NonCommercial-NoDerivatives 4.0 International (CC BY-NC-ND 4.0) License. 